\title{
Practices and thinking of laboratory detection in the aid to West Africa to fight against Ebola
}

\author{
Sun $\mathrm{Y}^{1}$, Zhao $\mathrm{XY}^{2}$, Zhang BK${ }^{1}$, Jiang $\mathrm{JF}^{1}$, Lu HJ ${ }^{1}$, Cao $\mathrm{YX}^{1}$, Wu GZ ${ }^{3}$, Qian ${ }^{1}$, Sun $\mathrm{YS}^{2}$, Zeng $\mathrm{YJ}^{4}$
}

First Chinese Laboratory Team to Aid Sierra Leone. yjzeng@bjut.edu.cn

\begin{abstract}
BACKGROUND: The outbreak of Ebola virus disease in West Africa has brought great disaster to the people's health in affected countries. China dispatched first group of public health experts and medical staff to Sierra Leone in September 2014 to fight Ebola.

METHODS: To systematically collect huge amount of primary data, and to make analysis, draw conclusions and lessons in terms of six aspects, respectively as training before departure, local disease information, track of epidemic situation, transformation of temporary laboratory, detection of Ebola virus samples as well as assessment through single blind test.

RESULTS: 1) Our team has launched preparatory works in advance before going to Sierra Leone. 2) Malaria is the country's severest infectious disease. 3) Observation centers were overcrowded with large number of suspected cases being inspected, implying high risk of nosocomial infection. 4) A BSL-II laboratory with 3 work areas and 2 buffer areas was built, achieving several major functions within 6 days. 5) Confirmed by South African Raqqa laboratory, our detection accuracy reached $100 \%$. 6) At one week before return, the daily average sample amount of our team reached 66 cases and our detection capability was equivalent to that of USA. CONCLUSIONS: Successful experience from fighting against Ebola in Sierra Leone could be summarized as: 1) Optimized processes and scientific security measures are prerequisite to improving the detection ability. 2) The close collaboration between laboratory and observation center has created a new model of China's foreign aid. 3) Comprehensive information investigation and training lay a solid foundation for the successful completion of tasks (Tab. 1, Ref. 3). Text in PDF www.elis.sk.

KEY WORDS: Ebola, virus, laboratory detection, Sierra Leone.
\end{abstract}

\section{Introduction}

According to the report of the World Health Organization (WHO), by April 5th, 2015, the global outbreak of the Ebola epidemic in West Africa has been reported a total of 25515 cases, including 10572 cases of death. The epidemic first broke out in Guinea, Sierra Leone and Liberia, and then spread to other 10 countries and regions, including America, Britain and Spain. Among these Ebola-affected countries, the epidemic was most severe in Sierra Leone. China provided successively four rounds of emergency materials totaling 750 million yuan to the affected countries in West Africa, and sent China's first group of public health experts and medical personnel on September 16th, 2014, to Sierra Leone to fight against Ebola. This paper retrospected and summarized the Ebola virus detection works by Chinese laboratory team in Sierra Leone.

${ }^{1}$ First Chinese Laboratory Team to Aid Sierra Leone, ${ }^{2}$ Center for Disease Control and Prevention of PLA, ${ }^{3}$ National Institute for Viral Disease Control and Prevention, Chinese Center for Disease Control and Prevention, and ${ }^{4}$ Beijing University of Technology, 100 Pingleyuan, Chaoyang District, Beijing, China.

Address for correspondence: Sun YS, Center for Disease Control and Prevention of PLA, China. Beijing University of Technology, 100 Pinalevuan, Chaoyang District, Beijing.

\section{Background of the task}

On August 1st, 2014, Fengfuzhen Chen, director-general of WHO, appealed to the international community and governments to provide full support for the fight against Ebola epidemic in West Africa. She also called the leaders of China, hoping that China could play a greater role in this fight. Guinea, Sierra Leone and other African countries issued formal invitations successively, requesting China to send medical aid. After several rounds of discussion in the meeting regarding the joint prevention and control of infectious diseases, China decided to send a laboratory team to Sierra Leone first. On September 16th, the laboratory team constituted by Chinese Center for Disease Control and Prevention (CDC China) and other 4 institutions, along with the PLA medical team organized by the 302 Military Hospitals of China, went to Sierra Leone to detect Ebola virus and operate the observation center, fighting against Ebola with the people of Sierra Leone.

\section{Main approach}

\section{Training before departure}

Our team had launched preparatory works in advance before going to Sierra Leone. On August 20th, the first laboratory team assembled quickly in a training base in Beijing to receive a 3-week 
Tab. 1. Sources of samples detected in Chinese laboratory.

\begin{tabular}{lccccc}
\hline Area & Total & Negative & Suspect & Positive & $\begin{array}{c}\text { Positive } \\
\text { rate (\%) }\end{array}$ \\
\hline BO & 2 & 0 & 1 & 1 & 50 \\
Bonthe & 1 & 1 & 0 & 0 & 0 \\
Kambia & 59 & 27 & 2 & 30 & 50.8 \\
Koinadugu & 11 & 6 & 2 & 3 & 27.3 \\
Kono & 1 & 1 & 0 & 0 & 0 \\
Moyamba & 1 & 0 & 0 & 1 & 0 \\
Tonkolili & 10 & 9 & 0 & 1 & 10 \\
Bombabli & 49 & 31 & 1 & 17 & 29.1 \\
Port Loko & 297 & 75 & 8 & 214 & 72.1 \\
Western Area & 1204 & 576 & 48 & 580 & 48.2 \\
\hline Total & 1635 & 726 & 62 & 847 & 51.8 \\
\hline
\end{tabular}

Statistics by November 12th, 2014

training course. For the uncertainty, suddenness and urgency of the task, we focused on the training of multiple skills for every member. According to strict, high-challenge requirements and field conditions, training was carried out by stages to make each member gain many practical skills. More than 20 experts and professors were invited to conduct systematic training on theory and skills, and make the team fully understand the local customs and epidemic situation of West Africa. Based on the standards issued by WHO and China's experiences in the prevention and control of SARS and H1N1 Flu, the standard operating procedures (SOPs) of laboratory testing were discussed and revised repeatedly. The revised SOPs were evaluated and validated in the National Institute for Viral Disease Control and Prevention, China CDC. More than 20 schemes and standby schemes of nearly 100 thousand characters were formulated on political security, biosecurity and administration security. All procedures and plans were elaborated to avoid any possible mistakes. Close attention was paid to comprehensive medical drills, which were conducted for the whole team for 3 times. According to the local climate, environment, epidemic status and other actual needs, the technical equipments and supplies to be carried were discussed and finalized one week before departure. More than 1000 kinds of medical and logistic supplies, totaling 170,000 sets (pieces) and 108 tons were packaged overnight and delivered to the airport on time.

\section{Local disease information}

Sierra Leone is one of the world's least developed countries, the GDP ranking the fourth from the end in the world. The infrastructure construction lags behind, with extensive shortage of supplies. According to the Ministry of Health and Sanitation, in addition to the epidemic of Ebola hemorrhagic fever, malaria is the country's severest infectious disease, accounting for $25 \%$ of total cases in 2012. The main malaria vectors are Anopheles gambiae, Anopheles funestus and Anopheles melas, and all cases are infected by Plasmodium falciparum. Other insect-borne and natural-focus diseases include lassa fever, yellow fever, dengue fever (caused by Sylvatic DENV strains), rabies and myiasis. Besides, Cholera, typhoid fever/paratyphoid fever, amebic dysentery, AIDS, tuberculosis, leprosy and infectious conjunctivitis are also common infectious diseases in Sierra Leone.

\section{Track of epidemic situation}

As soon as our team arrived at Sierra Leone, a contact was established quickly with the Ministry of Health and Sanitation under the coordination of the embassy. The national epidemic dynamics was closely traced every day, providing scientific guidance to our work. The Ebola epidemic in Sierra Leone was mainly introduced from Guinea, and the 12 administrative areas in the country were all affected by Ebola epidemic. The areas bordering on Guinea such as Kailahun and Kenema were the most severely affected areas in the early outbreak. With the development of the epidemic, the Western Area where the capital Freetown and its suburbs are located gradually became the most serious epidemic area. According to epidemic data announced by Sierra Leone Ministry of Health and Sanitation, by September 18th, 2014, the country had reported more than 1700 of confirmed cases and over 500 cases of death. The national external traffic was interrupted. Medical institutions faced a serious lack of facilities, and observation centers were overcrowded with large number of suspected cases being inspected, implying high risk of nosocomial infection. The local medical institution informed that 4 health care workers from USA had been infected and 20 nurses from Sierra Leone epidemic prevention team had died due to infection with Ebola virus. The situation of national disease control and prevention was very grim.

\section{Transformation of temporary laboratory}

In order to carry out detection work as soon as possible to resolve the major difficulty of scarce detection capacity in the local prevention and control system, our team was quickly acquainted with the facilities and overall layout of the hospital and proposed laboratory schemes after arriving Sierra Leone-China Friendship Hospital Firstly, based on Sierra Leone-China Friendship Hospital, common laboratory was transformed into temporary laboratory of biosafety level II (BSL-II) to completely separate people and materials. Our team seized every minute and second to investigate the site and draw sketches, and the transformation plan was determined in just one day. Moreover, we discussed the plan with the experts of American Galvestion National Laboratory and American CDC. Under the support of local Chinese-funded institutions, the laboratory was reconstructed within 6 days, involving the physical partition, lighting, water and power supply and biosafety equipment installation. A BSL-II laboratory with 3 work areas and 2 buffer areas was built, achieving several major functions: PCR detection, sample preservation, high temperature sterilization, preservation of undetected samples, pollutant disinfection, regional monitoring and equipment storage. It became a fixed facility that can support our mobile BSL-III laboratory, and all the preparatory works for detection can be carried out under emergency conditions.

In order to prevent the cross contamination in the detection area by Ebola patients from observation center, we set isolation board in the cross. Disinfection measures were taken in possibly contaminated areas, and regular disinfection was conducted in work area and living area, so as to minimize the risk of biosafety 1 . 


\section{Assessment through single blind test}

Strict requirements have been made by WHO for access of international laboratory to aid Africa, which must pass the comparison of laboratory detection and only qualified ones were allowed to go into operation. On September 24th, 5 blind samples from the WHO network laboratory (Raqqa Laboratory of South Africa) were detected in our newly transformed laboratory. We carried out the detection using 4 kinds of Ebola virus detection kits developed domestically, and the results were 4 positive and 1 negative. Confirmed by South African Raqqa laboratory, our accuracy reached $100 \%$. This is the first time for our self-developed Ebola detection kit to accept real virus verification. This result also confirms that the research principle and methods of domestic diagnostic reagents are scientific and rigorous, despite the absence of real viruses.

\section{Detection of Ebola virus samples}

Laboratory testing is an important part of the control and prevention of infectious diseases, accurate and fast detection is required in the premise of biosafety. Before our team arrived, only three testing laboratories from USA, South Africa and Canada were established. The average daily detection amounts were around 120 cases, which could not meet the demand of newly suspected cases. The international counterparts of each laboratory were facing great pressure of sample detection.

Our team began to receive samples from Sierra Leone on September 28th, on which 24 cases were tested, exceeding the aim of 20 cases/day set before going abroad. On October 23rd, the number of tested samples in four laboratories from China, South Africa, USA and Canada was 69, 55, 36 and 20, respectively, totaling 180 samples. The sample testing amount of our team came to the first among the four countries for the first time. Two breakthroughs in our team were made on October 31, i.e., the daily testing amount reached 106 cases and wasthe first again (USA, Britain, Canada and South Africa: 70, 46, 11 and 0 cases, respectively). On the same day, the total number of samples tested exceeded 1000, being 1096 cases. As the capability of testing steadily improved, our laboratory gained increasingly more respect from international colleagues. At the beginning of November, the British Ebola detection laboratory started to run officially, which further improved the testing capability in Sierra Leone.

Statistics issued in the website of Sierra Leone Ministry of Health and Sanitation show that a total of 1635 Ebola virus samples were tested by our team during the aid period. These samples came from over 20 treatment centers, observing centers, urban hospitals and community care units in 10 areas of Sierra Leone (See Table 1 for sample distribution area), accounting for $1 / 4$ of total sample size in the same period. The number of positive samples were 847 , accounting for $1 / 3$ of the total positive samples in Sierra Leone. The positive samples in our laboratory mainly came from the Western Area (including the capital), and over 1/2 of positive samples in this area were detected by our laboratory. At one week before return, the daily average sample amount reached 66 cases, second only 67 cases of USA laboratory over the same period. Our detection capability was equivalent to that of USA. The data above manifests the substantial contribution we have made for the control and prevention of Ebola epidemic in Sierra Leone.

\section{Experiences}

Optimized processes and scientific security measures are prerequisite to improving the detection ability

The work management processes were improved from the following four aspects. First, the communications with international laboratories were strengthened. According to the actual epidemic situation, the procedures of observation, sampling and sample delivery were formulated and standardized together with the personnel of Sierra Leone Ministry of Health and Sanitation in order to ensure the normalization, safety and reliability of sampling. Second, the internal tasks were refined, i.e., 6 processes including sample receiving, document verification, inactivation, RNA extraction, PCR detection and result evaluation and report were set and linked with each other closely. Third, the process was optimized technically. Clinical and epidemiological data are combined to validate and determine the main detection reagents and parallel detection standard. Disinfection Protocol of Mobile BSL-III Laboratory and Sample Inactivation Procedures have been revised, which greatly improved the detection efficiency and prolonged the service life of equipments. Fourth, the work shift system was established according to actual situation and the personnel combination was fixed. Through such measures as double batch and primary/secondary shift, the work load was allocated reasonably. Consequently, our detection capability was improved continuously.

The close collaboration between laboratory and observation center has created a new model of China's foreign aid

Our laboratory team and medical team immediately arrived at Sierra Leone in the most serious period of Ebola epidemic. The work was carried out rapidly relying on the close and seamless collaboration with Sierra Leone-China Friendship Hospital. Compared with other countries and international organizations, Chinese laboratory had more close connection with the observation center, and the process was more scientific, making the whole process integrated seamlessly from the receiving of suspected patients, sampling, sample delivery, detection, resulted feedback to the patient transfer for treatment. For example, besides the detection results of samples from all over the country would be reported to the Sierra Leone Ministry of Health and Sanitation in the same day, the result of samples from the observation center of 302 Military Hospital would be fed back as soon as possible. This greatly saved the time from the isolated observation of suspected patients to the diagnosis, transfer and treatment, and improved the transfer rate of suspected patients. Direct contribution was made to the performance of the second largest number of patients admitted and treated by Chinese observation center in Sierra Leone.

Comprehensive information investigation and training lay a solid foundation for the successful completion of tasks

The practice shows that the comprehensive information investigation of target area (including natural, geographical, cultural 
environment and epidemic information) in advance provided a powerful support for the smooth implementation of the task. Based on the specific local environment, our team prepared sufficient logistic equipment and medical supplies. In addition, we conducted a comprehensive training according to the collected information, so that all staff mastered the necessary knowledge and skills to perform tasks in a short period of time. After arrival, they could adapt to the local environment and working conditions quickly, and take measures as early as possible to avoid the risks expected.

\section{Special memory}

Unfortunately, during the two months in Sierra Leone, 7 medical colleagues working with us in the frontline combating Ebola died of Ebola virus infection. They are Godfree George, director of the National Hospital of Kambia area, Timasay, Liaison Officer in Sierra Leone-China Friendship Hospital accredited by the
Sierra Leone Ministry of Health and Sanitation, a local driver who delivered virus samples to Chinese laboratory every day, and 4 medical staff in Kingharman Road Government Hospital in which Chinese medical team was based. We deeply miss them, and are greatly proud of having combated the unprecedented Ebola epidemic with them.

\section{References}

1. National Health and Family Planning Commission of the People's Republic of China. Prevention and control scheme for Ebola hemorrhagic fever (Second Edition). Clin Educ Gen Practice 2014; 12 (5): 483-485.

2. Tao Zheng, Peitang Huang, Beifen Shen. Status and prospect of global biosecurity. Military Med Sci 2012; 36 (10): 721-724.

3. Fuchu He, Fusuo Gao. Biosecurity: commanding height of defense strategy. Qiushi 2014; (1): 53-54. 